\title{
Research on moving object tracking based on Markov model
}

\author{
Yao Can-rong ${ }^{1, a}$, Liu Shao-tao ${ }^{1, a}$ \\ ${ }^{1}$ School of Computer Science and Technology,Huaqiao University, Fujian Xiamen, 361021, China \\ ayaocanrong_hq@163.com
}

Key words: The tracking of the trajectory of the target;Hidden Markov;Vitby decoding

\begin{abstract}
The existing localization algorithms just only shows the static location features of the target, it can not be better to track to get the trajectory of the operation behavior in the mobile condition. This paper designs a trajectory target tracking algorithm based on Hidden Markov model. Firstly, based on the location information of the coverage area of the cell(including roads and subsection, the base station location) movement speed of the target, to construct the topology of actual highway for the restore problems of behavior trajectory, and calculate the transfer matrix between each section of highway; then, established a hidden Markov model; then, based on the Vitby decoding algorithm to take the most optimal solution of the probability of the best path and the best condition, to achieve the tracking of target, and then recover the target trajectory. The simulation results show that, the algorithm can accurately obtain motion track of the target in the resolution ratio of station cell.
\end{abstract}

\section{Introduction}

Wireless location technology based on signal detection heavily relies on radio propagation environment. To present a more accurate location results of estimation, it needs that the send and receive ends can realize the signal line of sight (LOS) propagation, and it needs the participation of multiple control platform, the strict synchronization of time is needed between different control platform . In order to improve the accuracy of positioning effectively, the domestic and foreign scholars have proposed many effective methods to inhibit or reduce the effects of NLOS . But every method has its applying occasions and assumptions, and the computational complexity is high, it is difficult to apply to the actual positioning system. Especially in the the geographical environment with complicated electromagnetic environment, the traditional radio propagation model and NLOS distribution model of cannot be used, the positioning scheme based on the matching of database location information showing its potential advantages. In literature , H. K. Lee et al proposed a location method by establishing the database of NLOS error to reduce the effect of NLOS on the measured data. Literature briefly summarized and gave the multiple correlated matching method for positioning method relating with database. Literature proposed a method by using the train of counter propagation neural network to establish a fingerprint database. The literature mainly analyzes the sources of the location error

\section{The establishing of model}

According to the model that track the target trajectory in scope of the community as shown in Figure 1, there are a number of paths under the coverage area. Compartmentalizing the road according to the condition of the cell coverage, such as upper half part of longitudinal road is completely covered by the base station $\mathrm{C}$, the next half is covered by three base station, so marking the longitudinal road as "1" and "2". 


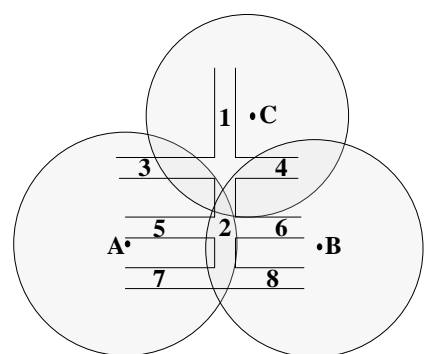

Figure. 1The model of target trajectory tracking

To describe Figure 1 by using hidden Markov model [13]. Making the road after subsection constitute the state set that contains $\mathrm{N}$ elements, and the set denoted as:

$S=\left\{s_{1}, s_{2}, \ldots, s_{N}\right\}$

The observation set is composed of the identification of base station and movement speed, the set denoted as:

$Y=\left\{o, v \mid o_{t_{i}} \in\left\{o_{1}, o_{2}, \ldots, o_{M}\right\}, v_{\min } \leq v \leq v_{\max }\right\}$

Among them there are $\mathrm{M}$ base stations $\left\{o_{1}, o_{2}, \ldots, o_{M}\right\}$, the mobile speed in the interval of $\left(v_{\text {min }}, v_{\text {max }}\right)$. The transfer probabilities from state ${ }^{s_{i}}$ to ${ }^{s_{j}}$ are:

$P\left(s_{j} \mid s_{i}\right)=a_{i j}, i=1,2, \ldots, N, j=1,2, \ldots, N$

In the state ${ }^{s_{i}}$, the observed conditional probability density function is:

$P\left(o_{k}, v \mid s_{i}\right)=b_{i}\left(o_{k}, v\right), k=1,2, \ldots, M, v_{\min } \leq v \leq v_{\max }$

Therefore, the target tracking problem can be attributed to that in the condition of the known observation probability $b_{i}\left(o_{k}, v\right)$, the transferring probability ${ }^{a_{i j}}$ and the observation set $\left\{y_{t_{i}}, y_{t_{2}}, \ldots, y_{t_{r}}\right\}$, $y_{t_{i}} \in Y$ to solve the state sequence $\left\{s_{t_{i}}, s_{t_{2}}, \ldots, s_{t_{t}}\right\}$.

\section{The target trajectory tracking algorithm based on Hidden Markov model}

It can be divided into three steps to achieve target tracking trajectory algorithm based on hidden Markov model: 1) topology construction; 2) establishing hidden Markov model; 3) optimal solution calculation. Firstly, based on the location information of cell coverage area(including roads and subsection, the base station location) and the mobile speed of users to construct the topology of the actual highway, determining the distribution of the initial state, the distribution matrix of the state transition and the probability density vector of observation process; then, establishing hidden Markov model; finally, to achieve the target tracking based on Vitby decoding algorithm.

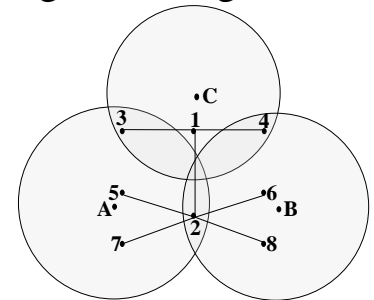

Figure 2 The graph structure that sections as node

\subsection{The topology construction}

The first step is the preparation stage, mainly constructing the topological graph of actual highway and calculating the transfer matrix between each section of highway.

Constructing the topology. Firstly, turning the highway distribution covered by base station shown in Figure 1 into graph structure shown in Figure 2, every section of the highway expressed by nodes.

. As shown in Figure 2, in the coverage area of the three base stations has 8 nodes, expressed the 8 nodes with 8 states $s_{i}$ (which $i=1,2, \ldots, 8$ ), representing the 8 section of highway in Figure 1. 
Set the transition probability matrix. There exist transition probability between the 8 points is $P\left(s_{j} \mid s_{i}\right)=a_{i j}$. As shown in Figure 2, the transition probability of the only two roads that having link is $P\left(s_{1} \mid s_{3}\right)=1$. According to the actual situation to set the transfer probability of two road with a plurality links: for example, if the No.2 section is only to the No. 6 and No. 8 road and the traffic flow of No. 6 and No. 8 sections are the same, then we can set $P\left(s_{8} \mid s_{2}\right)=P\left(s_{6} \mid s_{2}\right)=1 / 2$. And so on, we can construct the transition probability matrix of all 8 nodes.

Set the conditional observation probability matrix. In practical application, we can observe the information of two aspects: moving speed of the current target(expressed as $v$ in algorithm) and the cell that the current target belongs to (expressed as ${ }^{o}$ in algorithm ). So, when the target is in a path (in a state ${ }^{s_{i}}$ ), the observed speed of the target and the cell it belongs to has a certain probability, for example, when the target is in No.2 section, which may exist in the cell A or cell B, so $P\left(o_{A} \mid s_{2}\right)=P\left(o_{B} \mid s_{2}\right)=1 / 2$; besides, when the target is in No.2 section and the speed is ${ }^{v}$, density function of its conditional probability is: $f\left(v \mid s_{2}\right)$. Therefore, to establish the conditional probability distribution matrix beforehand according to the actual situation of the two measurements.

\subsection{Calculation of optimal solution}

The third step is to calculate the optimal solution by using the Vitby algorithm. When completed all calculations of probability according to figure 3, we can also traceback by it, to find out every time the road (state)that the target in [13]. The probability distribution of the street that the target in in the initial time is the initial state distribution, denoted as $\pi_{n}, n=1,2, \ldots, N$, and having $\sum_{n=1}^{N} \pi_{n}=1$. If after $\mathrm{T}$ times observation $\left\{y_{t_{1}}, y_{t_{2}}, \ldots, y_{t_{r}}\right\}$, the state of the moment is $s_{i}, \quad t$ moment in the probability of the best path of $s_{i}$ in t moment is $\phi_{s_{i}}(t), i=1,2, \ldots, N$. The observation value set that observed in $t_{T+1}$ is ${ }_{t_{t+1}}$, and the probability of the state ${ }^{s_{j}}$ is:

$P\left(x_{t_{r}}=s_{i}, y_{t}, y_{t_{2}}, \ldots, y_{t_{t}}, y_{t_{t+1}}, x_{t_{r+1}}=j\right)$

$=P\left(x_{t_{T}}=s_{i}, y_{t_{1}}, y_{t_{2}}, \ldots, y_{t_{T}}\right) \cdot P\left(x_{t_{T+1}}=s_{j} \mid x_{t_{T}}=s_{i}\right) \cdot P\left(y_{t_{t+1}} \mid x_{t_{r+1}}=s_{j}\right)$

$=\phi_{s_{i}}(t) \cdot a_{i j} \cdot b_{j}\left(y_{t_{t+1}}\right)$

So, in the ${ }^{t_{T+1}}$ moment, the probability of the best path of the target in state ${ }^{s_{j}}$ is: $\phi_{s_{j}}(t+1)=\max _{s, v_{n, k} \leq v \leq v_{\max }}\left(\phi_{s_{i}}(t) \cdot a_{i j}\right) \cdot b_{j}\left(y_{t_{t+1}}\right)$

From ${ }^{t_{T}}$ moment to ${ }^{t_{T+1}}$ moment, the most likely state as the goal in is:

$\psi_{s, j}(t+1)=\underset{s, s S}{\arg \max }\left(\phi_{s_{i}}(t) \cdot a_{i j}\right)$

Carrying out the Viterbi decoding algorithm according to (6), (7), we can get the most likely position the target in of the moment ${ }^{t_{T}}$ and ${ }^{t_{T+1}}$, connecting these positions can constitute the tracking of the object trajectory.

\section{Experimental analysis}

The classification of samples shown in Figure 5. The eventual target trajectory as shown in figure 6.

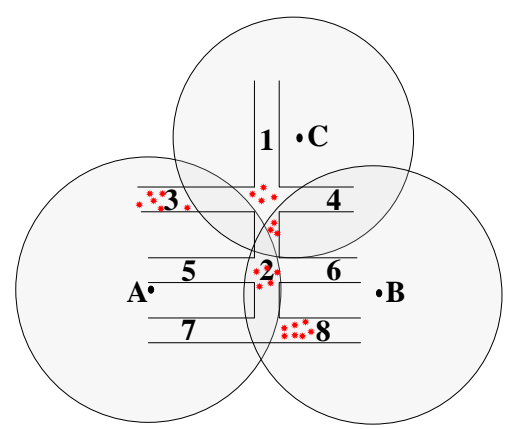

Figure 5 The distribution map of samples after Vitby decoding

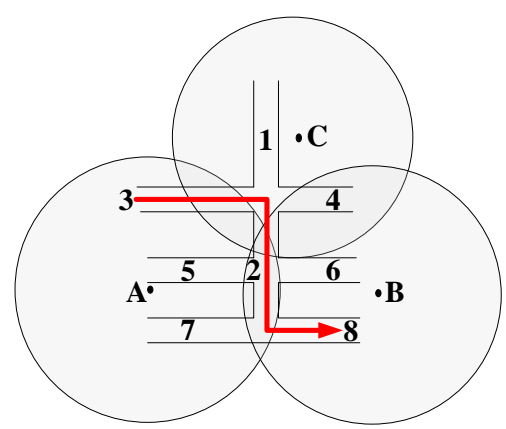

Figure 6 The final target trajectory diagram 
By using hidden Markov model can be better to track the target trajectory is, with the increasing of the observation points, the precision of the trajectory tracking will continue to increase. And the finer the division of the blocks, the better the precision of the trajectory tracking.

In Figure 7, if the observed velocity exists errors but the ownership information of observation area does not exist errors, the fitting parameters are $\mathrm{B} 0=-6.33, \mathrm{~B} 1=10.03$; if the observed velocity doesn't exist errors but the ownership information of observation area exists errors, the fitting parameters are $\mathrm{B} 0=-4.72, \mathrm{~B} 1=9.07$. From Figure 7 , we can know that the distribution trends in the two error conditions are similar in general, but the impact of observation velocity errors brought is small.

Figure 8 is a curve graph of probability of error of trajectory tracking on the basis of 20 sequences of 20 cell observation, every cell observing one time. In figure 8 , if the observed velocity exists errors but the ownership information of observation area does not exist errors, the fitting parameters are $\mathrm{B} 0=-6.21, \mathrm{~B} 1=9.98$; if the observed velocity doesn't exist errors but the ownership information of observation area exists errors, the fitting parameters are $\mathrm{B} 0=-4.66, \mathrm{~B} 1$ $=8.93$. Comparing Figure 7 with Figure 8 , we can know that, the increasing the length of observation sequence has little improvement on the tracking result of trajectory.

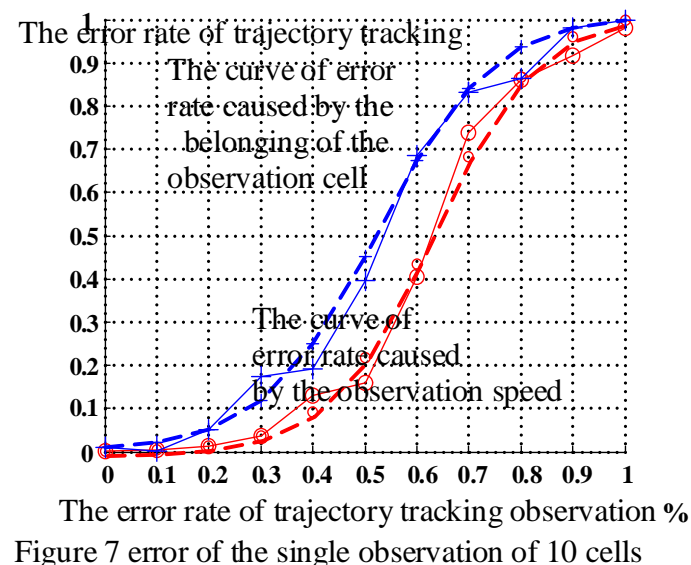

Figure 7 error of the single observation of 10 cells

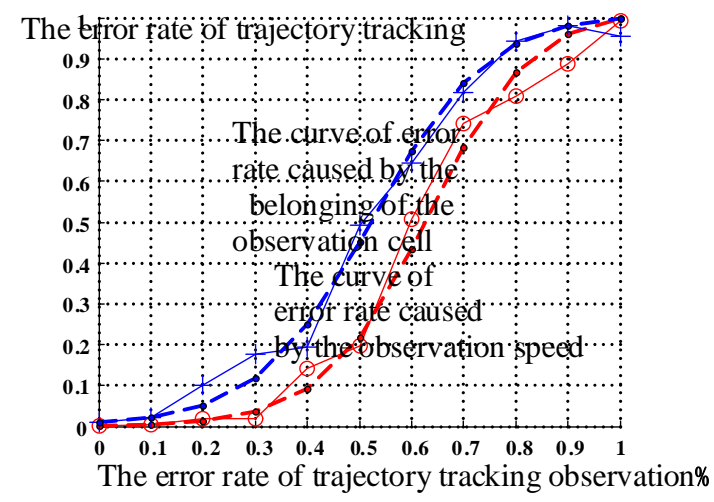

Figure 8 error of the single observation of 20 cells

\section{Conclusion}

In this paper, we design a kind of algorithm of target tracking based on Hidden Markov model aiming at the problem of the target trajectory tracking in the resolution of the base station cell. The algorithm firstly according to the information of geographic location and moving speed of user of the coverage area of cell to establish a hidden Markov model for the problem; then, based on the Vitby decoding algorithm to take the most optimal solution of the probability of the best path and the best condition. The simulation results show that, the algorithm can accurately obtain the track of the target in the resolution ratio of station cell.

\section{References}

[1] Y. Geng, J. Chen, K. Pahlavan, Motion detection using RF signals for the first responder in emergency operations: A PHASER project[C], 2013 IEEE 24nd International Symposium on Personal Indoor and Mobile Radio Communications (PIMRC), London,Britain Sep. 2013

[2] S. Li, Y. Geng, J. He, K. Pahlavan,Analysis of Three-dimensional Maximum Likelihood Algorithm for Capsule Endoscopy Localization, 2012 5th International Conference on Biomedical Engineering and Informatics (BMEI), Chongqing, China Oct. 2012 (page 721-725)

[3] Y. Geng, J. He, H. Deng and K. Pahlavan, Modeling the Effect of Human Body on TOA Ranging for Indoor Human Tracking with Wrist Mounted Sensor, 16th International Symposium on Wireless Personal Multimedia Communications (WPMC), Atlantic City, NJ, Jun. 2013.

[4] Y. Geng, J. He, K. Pahlavan, Modeling the Effect of Human Body on TOA Based Indoor Human Tracking[J], International Journal of Wireless Information Networks 20(4), 306-317 